\title{
A New and Simple Risk Predictor of Contrast-Induced Nephropathy in Patients Undergoing Primary Percutaneous Coronary Intervention: TIMI Risk Index
}

\author{
Ahmet Kaya ${ }^{D},{ }^{1}$ Ahmet Karataş, ${ }^{2}$ Yasemin Kaya, ${ }^{3}$ Harun Düğeroğlu, ${ }^{3}$ Seçkin Dereli, ${ }^{4}$ \\ and Adil Bayramoğlu ${ }^{1}$ \\ ${ }^{1}$ Department of Cardiology, Ordu University Medical School, Ordu, Turkey \\ ${ }^{2}$ Department of Nephrology, Ordu University Medical School, Ordu, Turkey \\ ${ }^{3}$ Department of Internal Medicine, Ordu University Medical School, Ordu, Turkey \\ ${ }^{4}$ Department of Cardiology, Ordu State Hospital, Ordu, Turkey
}

Correspondence should be addressed to Ahmet Kaya; doktorahmetkaya@yahoo.com

Received 22 June 2018; Accepted 27 August 2018; Published 26 September 2018

Academic Editor: Anne Knowlton

Copyright (c) 2018 Ahmet Kaya et al. This is an open access article distributed under the Creative Commons Attribution License, which permits unrestricted use, distribution, and reproduction in any medium, provided the original work is properly cited.

Background. The thrombolysis in myocardial infarction risk index (TRI) was developed to estimate prognosis at the initial contact of the healthcare provider in coronary artery disease patients without laboratory parameters. In this study, we aimed to investigate the relationship of the baseline TRI and contrast-induced nephropathy (CIN) in patients with ST-elevation myocardial infarction (STEMI). Methods. A total of 963 consecutive STEMI diagnosed patients who underwent primary percutaneous intervention were included in the study. TRI was calculated using the formula "heart rate $\times($ age/10) 2/SBP" on admission. CIN was defined as an increase in serum creatinine concentration $\geq 25 \%$, 48 hours later over the baseline. Results. Of the total of 963 patients, CIN was observed in $13 \%(n=128)$. TRI was significantly higher in the CIN $(+)$ group compared with the CIN $(-)$ group $(32.9 \pm 18.8$ vs $19.9 \pm 9.9, P<0.001)$. There was a stronger correlation between CIN and age, diastolic blood pressure, heart rate, Killip class, left ventricular ejection fraction, amount of contrast media, and diabetes mellitus. The amount of contrast media (OR $1.010,95 \% \mathrm{CI}$ $1.007-1.012, P<0.001$ ) and TRI (OR 1.047, 95\% CI 1.020-1.075, $P=001$ ) were independent predictors of CIN. The best threshold TRI for predicting CIN was $\geq 25.8$, with a $67.1 \%$ sensitivity and $80.4 \%$ specificity (area under the curve (AUC): $0.740,95 \%$ CI: $0.711-0.768, P<0.001)$. Conclusion. TRI is an independent predictor of CIN, and it may be used as a simple and reliable risk assessment of CIN in STEMI patients without the need for laboratory parameters.

\section{Introduction}

Contrast nephropathy (CIN) is characterized by an acute disruption in renal functions following exposure to contrast agents, and different studies have reported its incidence as $5-25 \%[1,2]$. Many factors are associated with the development of CIN, including advanced age, increased amount of contrast agent, basal renal failure, diabetes mellitus (DM), and hypertension (HT) [3]. It may develop more commonly after primary percutaneous intervention. Studies have shown that contrast nephropathy causes prolongation of hospitalization duration, increased in-hospital complications, and increased
1 -year mortality $[4,5]$. Early and correct recognition of CIN is crucial in the prevention of progression and improvement of outcomes [6].

The thrombolysis in myocardial infarction risk index (TRI) is a simple risk score designed for using at initial presentation to predict mortality in STEMI patients; it does not include any laboratory variables. The TRI is derived from three readily available clinical variables and is calculated using the equation (heart rate $\times($ age/10) $2 /$ systolic blood pressure) $[7,8]$. TRI has been linked to mortality and morbidity in many cardiovascular diseases such as ST-elevation myocardial infarction (STEMI), pulmonary embolism, and acute heart failure [9]. 
Various laboratory parameters and scoring systems have shown to be successful at predicting the development of CIN [10]. In this study, we aimed to evaluate the effectiveness of TRI, which is a clinical score easy to calculate in STEMI patients undergoing primary percutaneous intervention.

\section{Materials and Method}

2.1. Study Population. A total of 963 patients who presented with the diagnosis of STEMI between September 2015 and January 2018 and underwent primary PCI (pPCI) were retrospectively included. Patients with known allergy against contrast agents, those presented with cardiogenic shock, patients using oral anticoagulants, those with hematologic disease, chronic inflammatory or autoimmune disease, patients with a creatinine clearance $<60 \mathrm{~mL} / \mathrm{min}$, and patients with chronic renal failure who required dialysis were excluded from the study. Age, systolic blood pressure (SBP), and heart rate (HR) were obtained at the time of admission in all patients. For each patient, TRI score was calculated before PCI, and the relationship between TRI and the development of CIN was investigated. Patients' TRI was calculated using the formula "heart rate $\times($ age/10) $2 / \mathrm{SBP}$ " [7]. Blood samples for the full blood count and the biochemistry parameters were obtained at the time of admission and 48 hours later from all patients. Written or verbal informed consents were received from all patients, and the study protocol was approved by the hospital's local ethics committee in accordance with the Helsinki Declaration and Good Clinical Practice Guidelines.

2.2. Definitions. STEMI was defined as the presence of ST elevation at least $1 \mathrm{~mm}$ in two or more continuous leads ( $2 \mathrm{~mm}$ for V1-V3) or new-onset left bundle branch block. All patients underwent p-PCI within the first 12 hours after the onset of the chest pain. Before PCI, all patients were given acetylsalicylic acid (ASA) (300 mg) and clopidogrel $(600 \mathrm{mg})$ as well as unfractioned heparin (70 IU $/ \mathrm{kg}$ IV). After the intervention, all patients were given $1 \mathrm{mg} / \mathrm{kg}$ of subcutaneous enoxaparin twice daily, $100 \mathrm{mg} /$ day of ASA, and $75 \mathrm{mg} /$ day of clopidogrel. Patients who were using antihypertensive agents or those have a systolic blood pressure, which was measured at rest with five minutes intervals in different time periods higher than $140 \mathrm{mmHg}$ or a diastolic blood pressure higher than $90 \mathrm{mmHg}$, were considered hypertensive [11].

Patients who were using antidiabetics or having a postprandial blood glucose level higher than $200 \mathrm{mg} / \mathrm{dL}$ or fasting plasma glucose of at least two times above $126 \mathrm{mg} / \mathrm{dL}$ were considered diabetic [12].

Hyperlipidemia (HL) was defined as a total cholesterol higher than $200 \mathrm{mg} / \mathrm{dL}$, triglycerides level higher than $160 \mathrm{mg} / \mathrm{dL}$, and low-density lipoprotein level higher than $130 \mathrm{mg} / \mathrm{dL}$ [13]. Ejection fraction (EF) was calculated using the modified Simpson's method. For patients undergoing p-PCI, iopromide (Ultravist ${ }^{\circledR}$ ) was used as the nonionic iso- osmolar contrast agent. All patients were hydrated $(0.9 \%$ sodium chloride $1 \mathrm{~mL} / \mathrm{kg} /$ hour) via intravenous route for 12 hours after the intervention. Blood samples were drawn before and 48 hours after p-PCI for measurement of serum creatinine. The creatinine clearance was calculated by using the Cockcroft-Gault formula: (140-age) * (weight in $\mathrm{kg}) *$ $(0.85$ if female $) /(72 *$ creatinine $)$. CIN was defined as previously described and distinguished as grade 0 (serum creatinine increase $<25 \%$ above baseline and $<0.5 \mathrm{mg} / \mathrm{dL}$ above baseline), grade 1 (serum creatinine increase $\geq 25 \%$ above baseline and $<0.5 \mathrm{mg} / \mathrm{dL}$ above baseline), or grade 2 (serum creatinine increase $\geq 0.5 \mathrm{mg} / \mathrm{dL}$ above baseline) [14]. Preexisting chronic kidney disease (CKD) was defined as having an estimated glomerular filtration rate (GFR) value $<60 \mathrm{~mL} / \mathrm{min} / 1.73 \mathrm{~m}^{2}$.

2.3. Statistical Analysis. The data analysis was conducted using SPSS (version 20.0, SPSS Inc., Chicago, IL, USA) and MedCalc statistical software (trial version 12.7.8, Mariakerke, Belgium). Continuous variables are expressed as the mean \pm standard deviation. Categorical variables were compared using Chi-square or Fisher's exact tests and summarized as percentages. The Kolmogorov-Smirnov test was used to evaluate the distribution of the continuous variables. To predict CIN, age, gender, DM, HT, HL, systolic and diastolic blood pressure, heart rate, history of prior myocardial infarction (MI), Killip $\geq 3$, pre-MI medication, syntax score, TIMI flow, GFR, creatinine, contrast amount, and TRI were included in the univariate analysis. The parameters with $P<0.05$ were included in the multiple logistic analysis. Receiver operating characteristic (ROC) curves were used to predict the future incidence of CIN.

\section{Results}

In this study, a total of 963 patients were included (mean age $58.1+11.9$ and $77.3 \%$ male). A total of $128(13.3 \%)$ patients developed CIN. Among these, $7.6 \%$ had grade 1 CIN and $5.7 \%$ had grade 2 CIN. The clinical characteristics as well as the angiographic and PCI features of the findings are listed in Tables 1-3.

Age, gender, DM, HT, SBP, diastolic blood pressure (DBP), heart rate, Killip >2, EF, basal creatinine, basal GFR, amount contrast agent, syntax score, TIMI flow, and TRI were statistically different in the CIN-positive group compared with the CIN-negative group.

Among the significant parameters in the univariate analysis (age, gender, DM, HT, heart rate, EF, GFR, TIMI flow, syntax score, contrast amount, and TRI), those that were also found to be significant in the multiple regression analysis included contrast amount (odds ratio $(\mathrm{OR})=1.010$, 95\% confidence interval (CI): 1.007-1.012, $P<0.001)$ and TRI $((\mathrm{OR})=1.047,(\mathrm{CI}): 1.020-1.075, P<0.001)$ (Table 4$)$.

The best TRI for CIN prediction was found to be 25.8 in the ROC curve analysis (AUC: 0.740, 95\% CI: 0.711-0.768, $P<0.001)$. TRI $\geq 25.8$ predicted CIN development with $67.1 \%$ sensitivity and $80.4 \%$ specificity (Figure 1 ). 
TABLE 1: Comparison of baseline clinical characteristics of patients with and without CIN after PCI.

\begin{tabular}{|c|c|c|c|}
\hline Variables & CIN $(-)(n=835)$ & $\operatorname{CIN}(+)(n=128)$ & $P$ value \\
\hline Age, years & $56.8 \pm 11.4$ & $66.2 \pm 12.3$ & $<0.001$ \\
\hline Age $>75$ & $64(7.7)$ & $35(27.3)$ & $<0.001$ \\
\hline Gender, male, $(n, \%)$ & $660(79.0)$ & $84(65.6)$ & 0.001 \\
\hline Diabetes mellitus, $(n, \%)$ & $175(21)$ & $51(39.8)$ & $<0.001$ \\
\hline Hypertension, $(n, \%)$ & $333(39.9)$ & $65(50.8)$ & 0.020 \\
\hline Smoking, $(n, \%)$ & $328(39.2)$ & $45(35.2)$ & 0.191 \\
\hline Hyperlipidemia, $(n, \%)$ & $332(39.8)$ & $43(33.6)$ & 0.182 \\
\hline Family history of CAD, $(n, \%)$ & $193(23.1)$ & $32(25)$ & 0.725 \\
\hline Prior MI, $(n, \%)$ & $89(10.7)$ & $12(9.4)$ & 0.659 \\
\hline Prior PCI, $(n, \%)$ & $80(9.6)$ & $11(8.6)$ & 0.722 \\
\hline Prior CABG & $27(3.2)$ & $3(2.3)$ & 0.589 \\
\hline Systolic blood pressure, $\mathrm{mmHg}$ & $124 \pm 41$ & $131 \pm 25$ & 0.011 \\
\hline Diastolic blood pressure, $\mathrm{mmHg}$ & $77 \pm 14$ & $81 \pm 20$ & $<0.001$ \\
\hline Heart rate, $(\mathrm{p} / \mathrm{min})$ & $76 \pm 14$ & $80 \pm 20$ & $<0.001$ \\
\hline Killip class $(\geq \mathrm{II}),(n, \%)$ & $104(12.5)$ & $43(33.6)$ & $<0.001$ \\
\hline LV-EF, \% & $46.9 \pm 7.7$ & $43.2 \pm 8.8$ & $<0.001$ \\
\hline TRI & $32.9 \pm 18.8$ & $19.9 \pm 9.9$ & $<0.001$ \\
\hline
\end{tabular}

Values are expressed as mean \pm SD or percentages. CAD, coronary artery disease; MI, myocardial infarction; PCI, percutaneous coronary intervention; CABG, coronary artery bypass graft; LV-EF, left ventricular ejection fraction; TRI, the thrombolysis in myocardial infarction risk index.

TABLE 2: Comparison of biochemical and hematologic variables of patients with and without CIN after PCI.

\begin{tabular}{|c|c|c|c|}
\hline Variables & CIN $(-)(n=835)$ & CIN $(+)(n=128)$ & $P$ value \\
\hline Baseline creatinine, $\mathrm{mg} / \mathrm{dL}$ & $0.92 \pm 0.35$ & $1.09 \pm 0.51$ & $<0.001$ \\
\hline $72 \mathrm{~h}$ creatinine, $\mathrm{mg} / \mathrm{dL}$ & $0.99 \pm 0.25$ & $1.5 \pm 0.79$ & $<0.001$ \\
\hline$\Delta-\mathrm{Cr}$ & $0.06 \pm 0.02$ & $0.41 \pm 0.75$ & $<0.001$ \\
\hline Baseline GFR, $\mathrm{mL} / \mathrm{min} / 1.73 \mathrm{~m}^{2}$ & $88.6 \pm 23.8$ & $75.1 \pm 31.0$ & $<0.001$ \\
\hline Glucose, $\mathrm{mg} / \mathrm{dL}$ & $150 \pm 79$ & $186 \pm 102$ & $<0.001$ \\
\hline Hemoglobin, g/dL & $13.2 \pm 1.8$ & $13.0 \pm 2.0$ & 0.156 \\
\hline $\mathrm{CRP}, \mathrm{mg} / \mathrm{l}$ & $12.4 \pm 10.4$ & $17.7 \pm 14.7$ & $<0.001$ \\
\hline LDL-cholesterol, mg/dL & $115 \pm 34$ & $112 \pm 37$ & 0.328 \\
\hline HDL-cholesterol, mg/dL & $38 \pm 11$ & $37 \pm 11$ & 0.523 \\
\hline Triglyceride, $\mathrm{mg} / \mathrm{dL}$ & $138 \pm 84$ & $128 \pm 58$ & 0.191 \\
\hline
\end{tabular}

$\Delta$-Cr, increase of creatinine in 72 hours; GFR, glomerular filtration rate; CK-MB, creatinine kinase myocardial band; CRP, C-reactive protein; LDL, lowdensity lipoprotein; HDL, high-density lipoprotein.

TABle 3: Comparison of angiographic and treatment variables of patients with and without CIN after PCI.

\begin{tabular}{|c|c|c|c|c|}
\hline Variables & & CIN $(-)(n=835)$ & CIN $(+)(n=128)$ & $P$ value \\
\hline Amount of contrast media & & $232 \pm 66$ & $290 \pm 89$ & $<0.001$ \\
\hline \multicolumn{5}{|l|}{ Previous medications, \% } \\
\hline Acetylsalicylic acid & & 13.8 & 9.4 & 0.171 \\
\hline Statin & & 19.4 & 8,5 & 0.037 \\
\hline ACE inhibitors/ARB & & 19.5 & 14.4 & 0.192 \\
\hline Beta-blocker & & 5 & 4.7 & 0.868 \\
\hline Calcium channel blocker & & 5 & 8 & 0.267 \\
\hline Oral antidiabetic & & 15 & 21.9 & 0.047 \\
\hline Insulin & & 5.7 & 21.1 & $<0.001$ \\
\hline \multirow{5}{*}{ Infarct-related artery, $(n \%)$} & LM & $3(0.4)$ & $1(0.1)$ & \multirow{5}{*}{0.155} \\
\hline & LAD & $400(47.9)$ & $68(53.1)$ & \\
\hline & $\mathrm{LCx}$ & $132(15.8)$ & $10(7.8)$ & \\
\hline & RCA & $289(34.6)$ & $49(38.3)$ & \\
\hline & Other & $11(1.3)$ & - & \\
\hline Syntax score, $\%$ & & $15.4 \pm 6.8$ & $16.8 \pm 8.6$ & 0.035 \\
\hline TIMI flow $3,(n, \%)$ & & $775(92)$ & $101(79)$ & $<0.001$ \\
\hline
\end{tabular}

ACE, angiotensin-converting enzyme; ARB, angiotensin receptor blocker; LM, left main; LAD, left anterior descending artery; LCx, left circumflex artery; RCA, right coronary artery; TIMI, thrombolysis in myocardial infarction. 
TABLE 4: Independent predictors of contrast-induced nephropathy in multivariate logistic regression analysis.

\begin{tabular}{lcccc}
\hline & \multicolumn{2}{c}{ Univariate analysis } & \multicolumn{2}{c}{ Multivariate analysis } \\
& OR & $P$ value & OR & $95 \%$ CI \\
\hline Age & 1.071 & $<\mathbf{0 . 0 0 1}$ & 1.025 & $0.996-1.055$ \\
Sex & 0.506 & $<\mathbf{0 . 0 0 1}$ & 1.156 & $0.686-1.948$ \\
Diabetes mellitus & 0.400 & $<\mathbf{0 . 0 0 1}$ & 0.700 & $0.437-1.122$ \\
Hypertension & 0.643 & $\mathbf{0 . 0 2 0}$ & 0.888 & $0.553-1.426$ \\
Heart rate & 1.024 & $<\mathbf{0 . 0 0 1}$ & 1.007 & $0.992-1.023$ \\
LV-EF & 0.945 & $<\mathbf{0 . 0 0 1}$ & 0.986 & $0.958-1.015$ \\
TRI index & 1.071 & $<\mathbf{0 . 0 0 1}$ & 1.047 & $1.020-1.075$ \\
GFR & 0.978 & $<\mathbf{0 . 0 0 1}$ & 0.994 & $0.948-1.003$ \\
TIMI flow & 3.453 & $<\mathbf{0 . 0 0 1}$ & 1.556 & $0.830-2.918$ \\
Syntax score & 1.027 & $\mathbf{0 . 0 3 5}$ & 0.984 & $0.957-1.012$ \\
Amount of contrast media & 1.009 & $<\mathbf{0 . 0 0 1}$ & 1.010 & $1.007-1.012$ \\
\hline
\end{tabular}

TRI, the thrombolysis in myocardial infarction risk index; TIMI, thrombolysis in myocardial infarction; LV-EF, left ventricular ejection fraction; GFR, glomerular filtration rate.

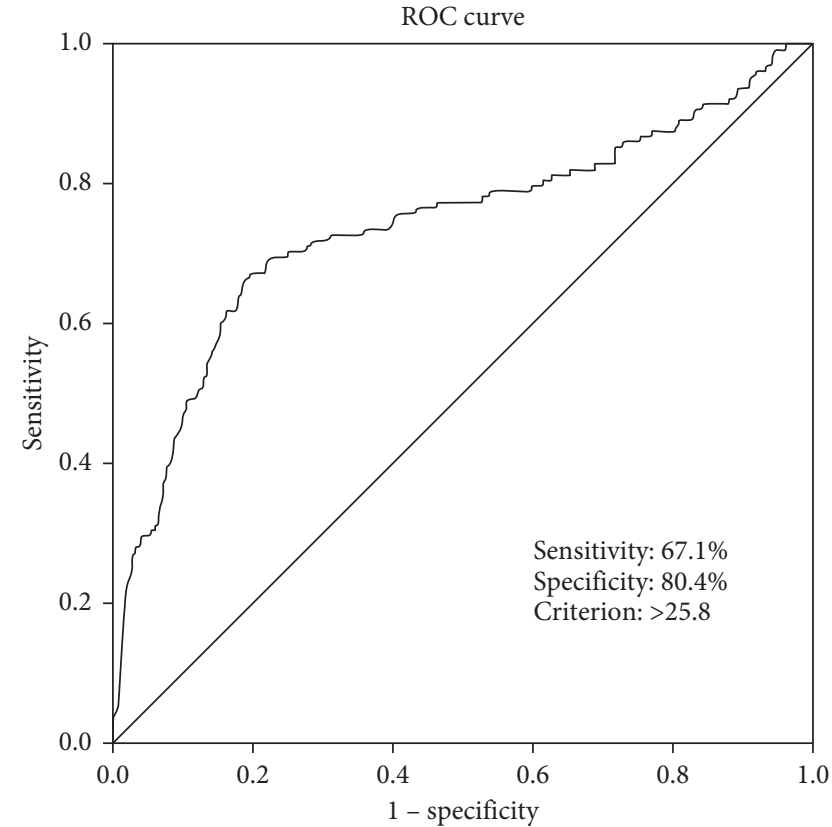

FIGURE 1: ROC curve analysis plot to determine the cut-off value of TRI in prediction of CIN.

\section{Discussion}

In our study, TRI was found to predict the development of contrast-induced nephropathy in STEMI patients who underwent PCI. It was found to be an independent predictor of CIN without the need for laboratory parameters.

CIN is a condition characterized by acute disruption in renal functions after exposure to contrast agents [15]. Medullary hypoxia due to renal artery vasoconstriction, direct cytotoxic effects of contrast agents, and decreased nitric oxide release secondary to the release of various mediators after the exposure are among the known pathophysiological mechanisms in the development of CIN [16]. In addition, protein precipitates due to inflammation secondary to renal medullary hypoxemia cause obstruction, playing a role in acute renal damage [17].
Previous studies have shown the association between the development of CIN and hospitalization duration, morbidity, and mortality [4]. Therefore, determination of the parameters that could predict the development of CIN before the intervention is of importance. Increased number of intervention because of the advancement in invasive cardiological techniques increases the risk for CIN [18]. Although various risk scores have been defined for prediction of the development of contrast-induced nephropathy after percutaneous coronary intervention (PCI), utility of these scores in clinical practice and their currency are limited [19, 20]. Especially, individual factors and characteristic variability of the populations on which the score systems are applied may have different effects on outcomes. Mehran's score is one of the most common risk scores in prediction of the risk for CIN [2]. In this scoring, a risk analysis is carried out with evaluation of many parameters. However, considering both numerical increase in the number of parameters and difficulties to take time for this in busy clinics, a risk score that can be applied more easily is warranted.

TIMI risk index is a score obtained by the formulation of age, systolic blood pressure, and heart rate, with a proven correlation with mortality and other outcome points in STEMI [7]. In a study, the relationship between TRI and long-term mortality and the development of heart failure in STEMI patients were demonstrated. In that study, although individual relationships of numerical values used in calculation of TRI (age, blood pressure, heart rate) were not showed, after the calculation this score was shown to be correlated with mortality [8]. Similarly, in our study, when separately evaluated age, systolic blood pressure, and heart rate were not significant predictors to indicate the development of CIN, while TRI was shown to independently predict the development of CIN. In many previous studies, various clinical factors have been shown to predict the development of CIN, although combined use of these parameters rather than individual use has been preferred for measurement of a score, and thus, their diagnostic value has been increased [21]. This suggests that better outcomes can be achieved by obtaining a few parameters using mathematical calculations. In a study, advanced age, anterior MI, 
and more amounts of contrast agent have been shown to be independently correlated with prediction of the development of CIN after p-PCI [22]. In another study, age has been shown as an independent predictor, although many studies have reported that SBP and heart rate were not independent predictors [9]. In our study, we found a significant correlation between the amount of contrast agents and development of CIN; age was not an independent predictor. In our study, it is not surprising to find a relationship between TRI, which contains pulse and systolic blood pressure that are the markers of hemodynamic changes and CIN. Previous studies have shown that disruption in renal blood flow causes acute renal damage with numerous neurohormonal mechanisms [5]. TRI provides ease of use with its features such as being a simple risk index used without a need for laboratory measurements and having only variables (age, heart rate, and systolic blood pressure).

The incidence of CIN has been reported to be higher compared with exposure to other elective contrast agents, because of the hemodynamic instability in primary percutaneous coronary intervention, the procedure itself, and the inability to take precautionary measure $[4,5]$. Considering the relation of CIN development with morbidity and mortality, it is important to identify particularly high-risk patients for new strategies that can be developed in the treatment of CIN. Therefore, as is shown in our study, the use of TRI which is easy to calculate in prediction of the development of CIN could be useful in identification of the patient group which could be evaluated in the development of new treatment strategies.

\section{Limitations}

This is an observational, single-institution study, which had a relatively small sample size and was thus subject to various unaccounted confounders inherent in such an analysis. Our findings should be confirmed and the application of the risk score validated in a large multicenter trial. Our patient group included only STEMI patients, and other patients with acute coronary syndrome or stable angina were not included.

\section{Conclusion}

In this study, we showed that increased TRI is an easily applicable simple and useful score without laboratory parameters. TRI is an independent early predictor of CIN in STEMI patients who are undergoing coronary intervention. Early prediction may provide time to prevent the progression of CIN and improve its negative impacts on outcome.

\section{Data Availability}

Data can be requested on an individual case basis via the corresponding author.

\section{Disclosure}

This paper has been presented as an abstract in the 14th International Congress of Update in Cardiology and Cardiovascular Surgery in The American Journal of Cardiology.

\section{Conflicts of Interest}

The authors have no conflicts of interest to declare.

\section{References}

[1] O. G. Shaker, A. El-Shehaby, and M. El-Khatib, "Early diagnostic markers for contrast nephropathy in patients undergoing coronary angiography," Angiology, vol. 61, no. 8, pp. 731-736, 2010.

[2] R. Mehran, E. D. Aymong, E. Nikolsky et al., "A simple risk score for prediction of contrast-induced nephropathy after percutaneous coronary intervention: development and initial validation," Journal of the American College of Cardiology, vol. 44, no. 7, pp. 1393-1399, 2004.

[3] R. Pappy, S. Stavrakis, T. A. Hennebry, and M. S. Abu-Fadel, "Effect of statin therapy on contrast-induced nephropathy after coronary angiography: a meta-analysis," International Journal of Cardiology, vol. 151, no. 3, pp. 348-353, 2011.

[4] T. Senoo, M. Motohiro, H. Kamihata et al., "Contrast-induced nephropathy in patients undergoing emergency percutaneous coronary intervention for acute coronary syndrome," American Journal of Cardiology, vol. 105, no. 5, pp. 624-628, 2010.

[5] P. A. McCullough, R. Wolyn, L. L. Rocher, R. N Levin, and W. W. Oneil, "Acute renal failure after coronary intervention: incidence, risk factors, and relationship to mortality," American Journal of Medicine, vol. 103, no. 5, pp. 368-375, 1997.

[6] T. Perrin, E. Descombes, and S. Cook, "Contrast-induced nephropathy in invasive cardiology," Swiss Medical Weekly, vol. 19, pp. 1-11, 2012.

[7] D. A. Morrow, E. M. Antman, R. P. Giugliano et al., "A simple risk index for rapid initial triage of patients with ST-elevation myocardial infarction: an InTIME II substudy," The Lancet, vol. 358, no. 9293, pp. 1571-1575, 2001.

[8] S. Wiviott, D. Morrow, P. Frederick et al., "Performance of the thrombolysis in myocardial infarction risk index in the national registry of myocardial infarction-3 and-4: a simple index that predicts mortality in ST-segment elevation myocardial infarction," Journal of the American College of Cardiology, vol. 44, no. 4, pp. 783-789, 2004.

[9] Q. Truong, C. Cannon, N. Zakai et al., "Thrombolysis in myocardial Infarction (TIMI) risk index predicts long-term mortality and heart failure in patients with ST-elevation myocardial infarction in the TIMI 2 clinical trial," American Heart Journal, vol. 157, no. 4, pp. 673-679.e1, 2009.

[10] A. Kaya, Y. Kaya, S. Topçu et al., "Neutrophil-to-lymphocyte ratio predicts contrast-induced nephropathy in patients undergoing primary percutaneous coronary intervention," Angiology, vol. 65, no. 1, pp. 51-56, 2013.

[11] J. A. Whitworth and J. Chalmers, "World Health Organisation-International Society of Hypertension (WHO/ ISH) hypertension guidelines," Clinical and Experimental Hypertension, vol. 26, no. 7-8, pp. 747-752, 2004.

[12] American Diabetes Association, "Diagnosis and classification of diabetes mellitus," Diabetes Care, vol. 37, no. 1, pp. S81-S90, 2014.

[13] S. M. Grundy, J. I. Cleeman, C. N. Merz et al., "Implications of recent clinical trials for the national cholesterol education program adult treatment panel III guidelines," Circulation, vol. 110, no. 2, pp. 227-239, 2004.

[14] K. J. Harjai, A. Raizada, C. Shenoy et al., "A comparison of contemporary definitions of contrast nephropathy in patients 
undergoing percutaneous coronary intervention and a proposal for a novel nephropathy grading system," American Journal of Cardiology, vol. 101, no. 6, pp. 812-819, 2008.

[15] D. Russo, R. Minutolo, B. Cianciaruso, B. Memoli, G. Conte, and L. De Nicola, "Early effects of contrast media on renal hemodynamics and tubular function in chronic renal failure," Journal of the American Society of Nephrology, vol. 6, no. 5, pp. 1451-1458, 1995.

[16] R. Solomon and H. L. Dauerman, "Contrast-induced acute kidney injury,” Circulation, vol. 122, no. 23, pp. 2451-2455, 2010.

[17] I. Goldenberg and S. Matetzky, "Nephropathy induced by contrast media: pathogenesis, risk factors and preventive strategies," Canadian Medical Association Journal, vol. 172, no. 11, pp. 1461-1471, 2005.

[18] R. Mehran, S. Brar, and G. Dangas, "Contrast-induced acute kidney injury. Underappreciated or a new marker of cardiovascular mortality?," Journal of the American College of Cardiology, vol. 55, no. 20, pp. 2210-2211, 2010.

[19] Y. M. Gao, D. Li, H. Cheng, and Y. P. Chen, "Derivation and validation of a risk score for contrast-induced nephropathy after cardiac catheterization in Chinese patients," Clinical and Experimental Nephrology, vol. 18, no. 6, pp. 892-898, 2014.

[20] N. Fu, X. Li, S. Yang et al., "Risk score for the prediction of contrast-induced nephropathy in elderly patients undergoing percutaneous coronary intervention," Angiology, vol. 64, no. 3, pp. 188-194, 2013.

[21] R. A. Abellás-Sequeiros, S. Raposeiras-Roubín, E. Abu-Assi et al., "Mehran contrast nephropathy risk score: is it still useful 10 years later?," Journal of Cardiology, vol. 67, no. 3, pp. 262-267, 2016.

[22] G. Marenzi, G. Lauri, E. Assanelli et al., "Contrast-induced nephropathy in patients undergoing primary angioplasty for acute myocardial infarction," Journal of the American College of Cardiology, vol. 44, no. 9, pp. 1780-1785, 2004. 


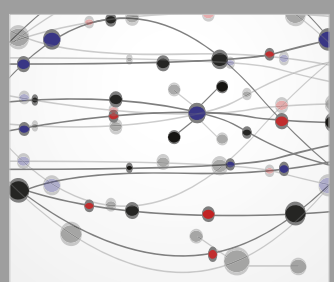

The Scientific World Journal
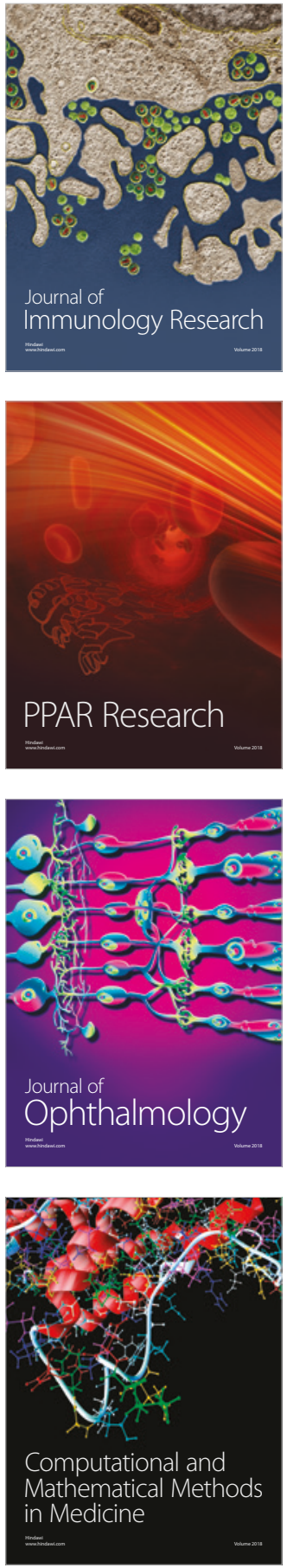

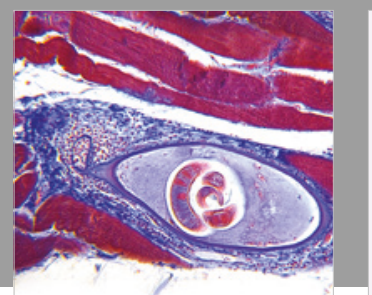

Gastroenterology Research and Practice

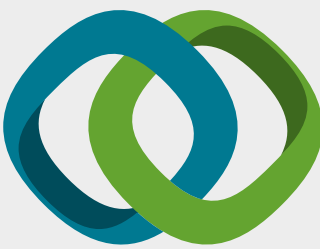

\section{Hindawi}

Submit your manuscripts at

www.hindawi.com
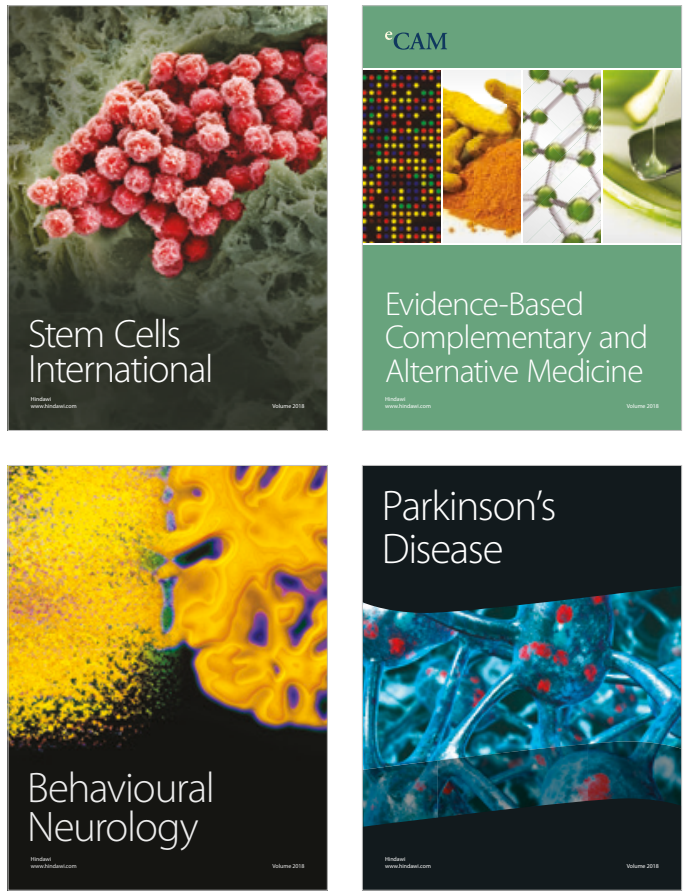

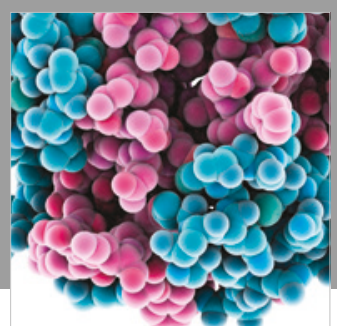

ournal of

Diabetes Research

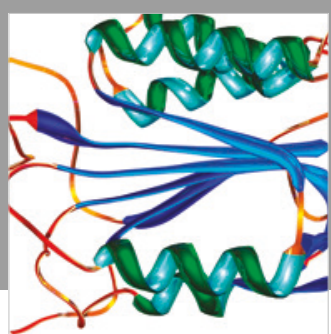

Disease Markers
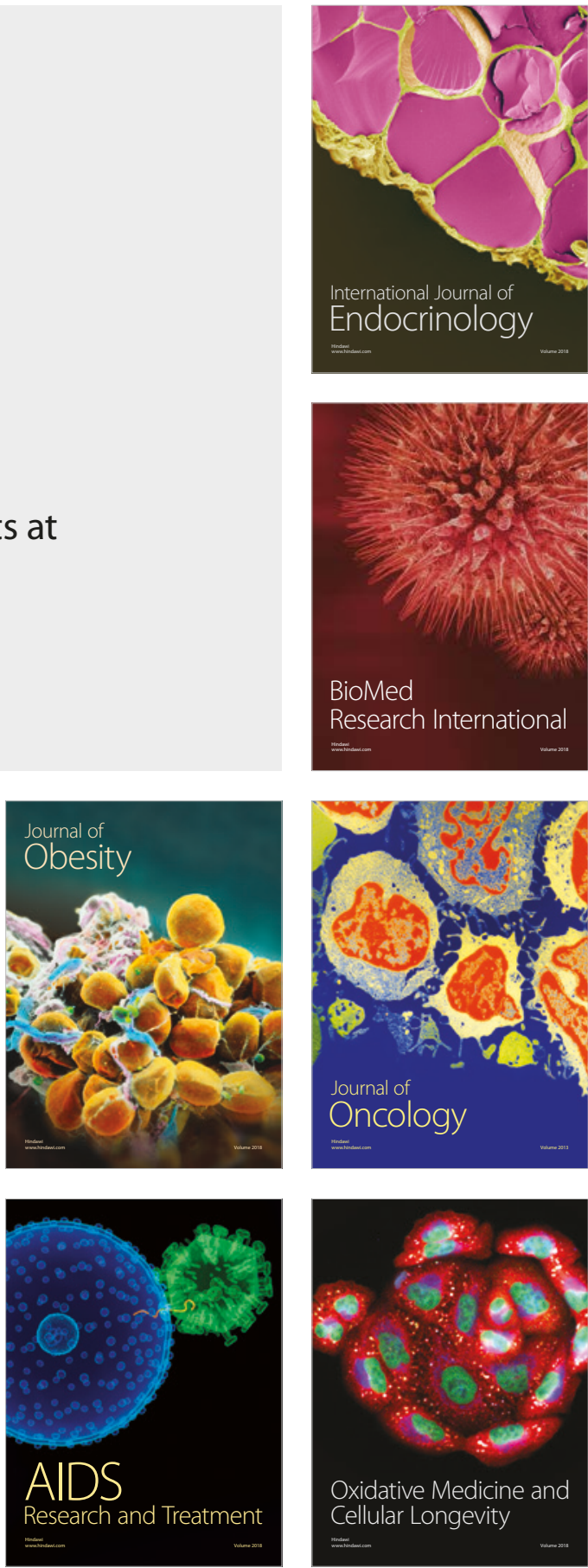